\title{
INTERNATIONALER HANDEL MIT DIENSTLEISTUNGEN
}

Der internationale Dienstleistungshandel gewinnt sowohl innerhalb des OECD-Raums als auch zwischen den OECDLändern und der übrigen Welt zunehmend an Bedeutung. Auf die traditionellen Dienstleistungen - Verkehrsleistungen, Versicherungsleistungen für den Warenhandel sowie den Reiseverkehr - entfällt etwa die Hälfte des internationalen Dienstleistungshandels, aber der Handel mit neueren Kategorien von Dienstleistungen, insbesondere solchen, die über das Internet abgewickelt werden können, expandiert rasch.

\section{Definition}

Der internationale Dienstleistungshandel wird gemäß dem Zahlungsbilanzhandbuch des Internationalen Währungsfonds (IWF) definiert. Dienstleistungen umfassen Verkehrsleistungen (Fracht- und Personenbeförderung), Fremdenverkehrsleistungen (hauptsächlich Ausgaben von Touristen und Geschäftsreisenden für Waren und Dienstleistungen), Kommunikationsleistungen (Post, Telefon, Satellitenübertragung usw.), Bauleistungen, Versicherungs- und Finanzdienstleistungen, EDV- und Informationsdienstleistungen, Tantiemen und Lizenzgebühren, sonstige Unternehmensdienstleistungen (Transithandel, Leasinggeschäfte, technische und freiberufliche Dienstleistungen usw.), Dienstleistungen für Kultur und Freizeit (Gebühren für Filmverleih, Gagen von Schauspielern und sonstigen Künstlern, aber ohne Erwerb von Filmen, Musikaufzeichnungen, Büchern usw.) sowie nicht in der vorstehenden Aufzählung enthaltene öffentliche Dienstleistungen.

\section{Vergleichbarkeit}

Die fünfte Auflage des IWF-Zahlungsbilanzhandbuchs (BPM5) erschien 1993 und wurde daraufhin nach und nach von den

\section{Überblick}

Zwischen 2008 und 2011 verzeichneten die Vereinigten Staaten den bei weitem höchsten Überschuss im Dienstleistungshandel, gefolgt vom Vereinigten Königreich, der Schweiz und Spanien.

2011 waren die Dienstleistungsausfuhren in den Vereinigten Staaten, dem Vereinigten Königreich, Deutschland und Frankreich am höchsten. Die Vereinigten Staaten waren in diesem Zeitraum der größte Importeur von Dienstleistungen, während das Vereinigte Königreich von Deutschland und Frankreich überholt wurde.

Im Durchschnitt des Dreijahreszeitraums bis Ende 2011 konnten nur vier Mitgliedsländer, nämlich Luxemburg, Estland, die Schweiz und Griechenland, Überschüsse im Dienstleistungshandel von über $5 \%$ des BIP verbuchen. Irland, Kanada, die Slowakische Republik und Mexiko verzeichneten für diesen Zeitraum Defizite von über 1\% des BIP.

Es ist zu beachten, dass das Gesamtdefizit Irlands im Handel mit Dienstleistungen von durchschnittlich $11,7 \%$ des BIP im Zeitraum 2000-2002 auf durchschnittlich 3,2\% des BIP im Zeitraum 2009-2011 gesunken ist, da die irischen Dienstleistungsausfuhren insbesondere auf Grund dynamischer Computerdienstleistungen rascher expandierten als die Dienstleistungseinfuhren.
Ländern angewandt. Alle OECD-Länder übermitteln die Daten über den internationalen Dienstleistungshandel jetzt weitgehend gemäß dem BPM5-Referenzrahmen. Die Daten für Australien werden gemäß dem neuen BPM6-Standard veröffentlicht. Bis Ende 2014 werden die meisten OECD-Länder von BPM5 auf BPM6 umgestiegen sein.

\section{Dienstleistungsbilanz: Ausfuhr von Dienstleistungen abzüglich Einfuhr von Dienstleistungen}

In Prozent des BIP

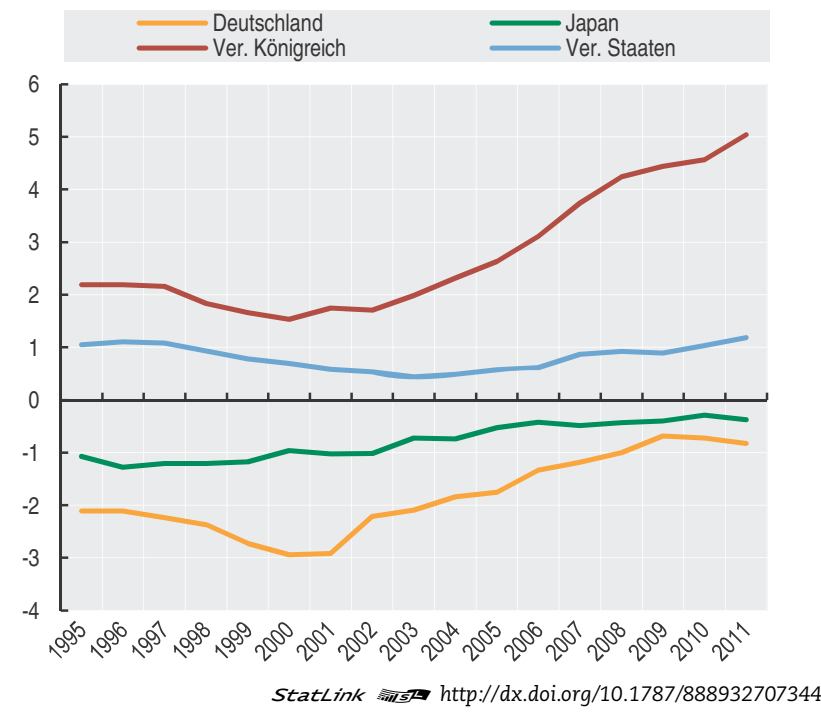

\section{Quelle}

- OECD (2012), Main Economic Indicators, OECD Publishing.

- OECD (2012), OECD Statistics on International Trade in Services, OECD Publishing.

\section{Weitere Informationen}

Analysen

- OECD (2012), Strategic Transport Infrastructure Needs to 2030, OECD Publishing.

\section{Statistiken}

- OECD (2012), International Trade by Commodity Statistics, OECD Publishing.

- OECD (2012), Statistics on International Trade in Services, OECD Publishing.

Zur Methodik

- Internationaler Währungsfonds (IWF) (2009), Balance of Payments and International Investment Position Manual, 6. Ausgabe, IWF, Washington D.C.

- OECD et al. (2010), Manual on Statistics of International Trade in Services, Vereinte Nationen.

\section{Websites}

- International Trade and Balance of Payments Statistics, www.oecd.org/std/trade-services.

- Services Trade Restrictiveness Index, www.oecd.org/trade/stri. 


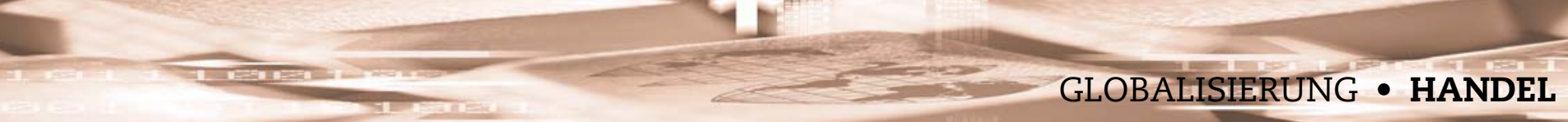

INTERNATIONALER HANDEL MIT DIENSTLEISTUNGEN

Internationaler Handel mit Dienstleistungen

Mrd. US-Dollar

\begin{tabular}{|c|c|c|c|c|c|c|c|c|c|c|c|c|}
\hline & \multicolumn{4}{|c|}{ Dienstleistungssaldo } & \multicolumn{4}{|c|}{ Einfuhren } & \multicolumn{4}{|c|}{ Ausfuhren } \\
\hline & 2000 & 2008 & 2010 & 2011 & 2000 & 2008 & 2010 & 2011 & 2000 & 2008 & 2010 & 2011 \\
\hline Australien & 0.5 & -4.0 & -3.5 & -9.2 & 19.3 & 47.9 & 51.1 & 60.9 & 19.8 & 44.0 & 47.6 & 51.7 \\
\hline Belgien & 2.1 & 5.0 & 8.2 & 4.7 & 32.3 & 83.0 & 82.4 & 89.8 & 34.3 & 88.1 & 90.6 & 94.5 \\
\hline Chile & .. & -1.2 & -1.8 & -2.4 & 4.8 & 11.9 & 12.6 & 14.8 & 4.1 & 10.7 & 10.8 & 12.4 \\
\hline Dänemark & 2.4 & 10.1 & 8.6 & 8.6 & 22.1 & 62.3 & 51.9 & 57.1 & 24.5 & 72.4 & 60.5 & 65.7 \\
\hline Deutschland & -55.0 & -37.4 & -24.2 & -31.6 & 138.2 & 292.8 & 267.7 & 296.5 & 83.2 & 255.4 & 243.5 & 265.0 \\
\hline Estland & 0.6 & 1.9 & 1.8 & 1.7 & 0.9 & 3.3 & 2.8 & 3.7 & 1.5 & 5.3 & 4.6 & 5.4 \\
\hline Finnland & -1.7 & 1.0 & 0.3 & 0.4 & 9.4 & 30.7 & 26.6 & 26.7 & 7.7 & 31.7 & 26.9 & 27.1 \\
\hline Frankreich & 17.2 & 24.1 & 21.0 & 33.7 & 65.7 & 141.0 & 171.2 & 191.0 & 82.8 & 165.1 & 192.2 & 224.7 \\
\hline Griechenland & 8.2 & 25.1 & 17.5 & 20.4 & 11.5 & 24.8 & 20.2 & 19.4 & 19.6 & 49.8 & 37.7 & 39.8 \\
\hline Irland & -13.0 & -11.2 & -8.8 & -2.5 & 31.4 & 110.6 & 107.2 & 115.8 & 18.4 & 99.3 & 98.4 & 113.3 \\
\hline Island & -0.1 & -0.3 & 0.3 & 0.3 & 1.2 & 2.4 & 2.2 & 2.6 & 1.0 & 2.1 & 2.5 & 2.9 \\
\hline Israel & 3.7 & 4.1 & 6.6 & 6.6 & 12.1 & 19.8 & 18.1 & 20.2 & 15.7 & 23.9 & 24.8 & 26.8 \\
\hline Italien & 1.1 & -12.6 & -12.0 & -9.1 & 55.4 & 127.8 & 110.8 & 116.1 & 56.5 & 115.2 & 98.8 & 107.0 \\
\hline Japan & -45.8 & -20.7 & -16.1 & -22.1 & 115.0 & 169.4 & 157.4 & 167.5 & 69.2 & 148.7 & 141.3 & 145.4 \\
\hline Kanada & -3.9 & -20.2 & -22.1 & -24.9 & 44.1 & 88.1 & 91.3 & 100.5 & 40.2 & 67.9 & 69.2 & 75.6 \\
\hline Korea & -2.0 & -5.7 & -8.6 & -4.4 & 33.6 & 96.4 & 95.9 & 99.4 & 31.5 & 90.6 & 87.3 & 95.0 \\
\hline Luxemburg & 6.8 & 29.2 & 30.0 & 32.0 & 13.2 & 39.0 & 36.7 & 41.1 & 20.0 & 68.0 & 66.7 & 73.1 \\
\hline Mexiko & -3.6 & -7.1 & -10.1 & -14.2 & 17.1 & 24.7 & 25.3 & 29.5 & 13.4 & 17.6 & 15.2 & 15.3 \\
\hline Neuseeland & -0.1 & -0.5 & -0.3 & -0.8 & 4.5 & 9.7 & 9.3 & 10.9 & 4.4 & 9.3 & 9.0 & 10.1 \\
\hline Niederlande & -2.1 & 13.0 & 10.5 & 13.5 & 51.4 & 92.2 & 85.2 & 94.0 & 49.3 & 105.2 & 95.7 & 107.5 \\
\hline Norwegen & 2.7 & 0.3 & -3.2 & .. & 15.0 & 44.5 & 42.9 & .. & 17.8 & 44.8 & 39.7 & \\
\hline Österreich & 6.5 & 20.8 & 17.4 & 19.5 & 16.5 & 42.8 & 37.0 & 42.4 & 23.0 & 63.4 & 54.5 & 61.8 \\
\hline Polen & 1.4 & 5.0 & 3.1 & 6.1 & 9.0 & 30.5 & 29.6 & 31.0 & 10.4 & 35.5 & 32.7 & 37.0 \\
\hline Portugal & 2.0 & 9.7 & 8.9 & 10.8 & 7.0 & 16.5 & 14.4 & 15.9 & 9.1 & 26.1 & 23.3 & 26.7 \\
\hline Schweden & -1.5 & 16.6 & 17.8 & 20.4 & 24.6 & 53.8 & 47.7 & 55.2 & 23.1 & 70.2 & 65.3 & 75.6 \\
\hline Schweiz & 17.9 & 46.3 & 48.8 & 56.0 & 12.8 & 30.8 & 34.7 & 40.7 & 30.7 & 77.1 & 83.4 & 96.5 \\
\hline Slowak. Rep. & 0.4 & -0.7 & -1.0 & -0.5 & 1.8 & 9.2 & 6.8 & 7.1 & 2.2 & 8.5 & 5.8 & 6.6 \\
\hline Slowenien & 0.5 & 2.1 & 1.7 & 2.0 & 1.7 & 5.2 & 4.4 & 4.7 & 2.2 & 7.2 & 6.1 & 6.7 \\
\hline Spanien & 19.4 & 37.7 & 36.4 & 47.6 & 33.2 & 105.4 & 88.0 & 94.6 & 52.6 & 142.8 & 124.3 & 142.2 \\
\hline Tschech. Rep. & 1.4 & 4.3 & 3.9 & 3.8 & 5.4 & 17.4 & 17.0 & 19.4 & 6.9 & 21.8 & 20.9 & 23.1 \\
\hline Türkei & 11.4 & 17.7 & 15.5 & 18.3 & 8.1 & 17.8 & 19.3 & 20.7 & 19.5 & 33.1 & 34.7 & 39.0 \\
\hline Ungarn & 0.8 & 2.3 & 3.9 & 4.5 & 4.8 & 18.0 & 15.5 & 17.2 & 5.6 & 20.2 & 19.4 & 21.8 \\
\hline Ver. Königreich & 22.6 & 111.8 & 103.5 & 122.4 & 101.9 & 215.1 & 177.9 & 188.0 & 124.7 & 324.9 & 281.4 & 310.3 \\
\hline Ver. Staaten & 69.0 & 131.8 & 150.4 & 178.5 & 219.0 & 403.4 & 403.2 & 427.4 & 288.0 & 535.2 & 553.6 & 606.0 \\
\hline EU27 & .. & .. & .. &.. & .. & .. & .. &.. & .. & .. & .. & .. \\
\hline OECD & 67.1 & 396.1 & 405.2 &.. & 1143.6 & 2492.4 & 2363.8 & .. & 1210.2 & 2889.5 & 2769.2 & .. \\
\hline Brasilien & -7.2 & -16.7 & -30.8 & -37.9 & 16.7 & 47.1 & 62.6 & 76.3 & 9.5 & 30.5 & 31.8 & 38.4 \\
\hline China & -5.6 & -11.8 & .. &.. & 36.0 & 158.9 & .. &.. & 30.4 & 147.1 &.. & .. \\
\hline Indien & -2.5 & 19.5 & 6.9 & .. & 19.2 & 88.0 & 116.7 & .. & 16.7 & 107.5 & 123.6 & .. \\
\hline Indonesien & -10.4 & -13.0 & -9.3 & -11.8 & 15.6 & 28.2 & 26.1 & 32.4 & 5.2 & 15.2 & 16.8 & 20.5 \\
\hline Russ. Föderation & -6.7 & -24.3 & -28.7 & -35.9 & 16.2 & 75.5 & 73.7 & 90.0 & 9.6 & 51.2 & 45.0 & 54.0 \\
\hline Südafrika & -0.8 & -4.2 & -4.5 & -4.8 & 5.8 & 17.0 & 18.5 & 19.7 & 5.0 & 12.8 & 14.0 & 14.8 \\
\hline
\end{tabular}

StatLink AोIs http://dx.doi.org/10.1787/888932707306

\section{Dienstleistungsbilanz: Ausfuhr von Dienstleistungen abzüglich Einfuhr von Dienstleistungen} In Prozent des BIP

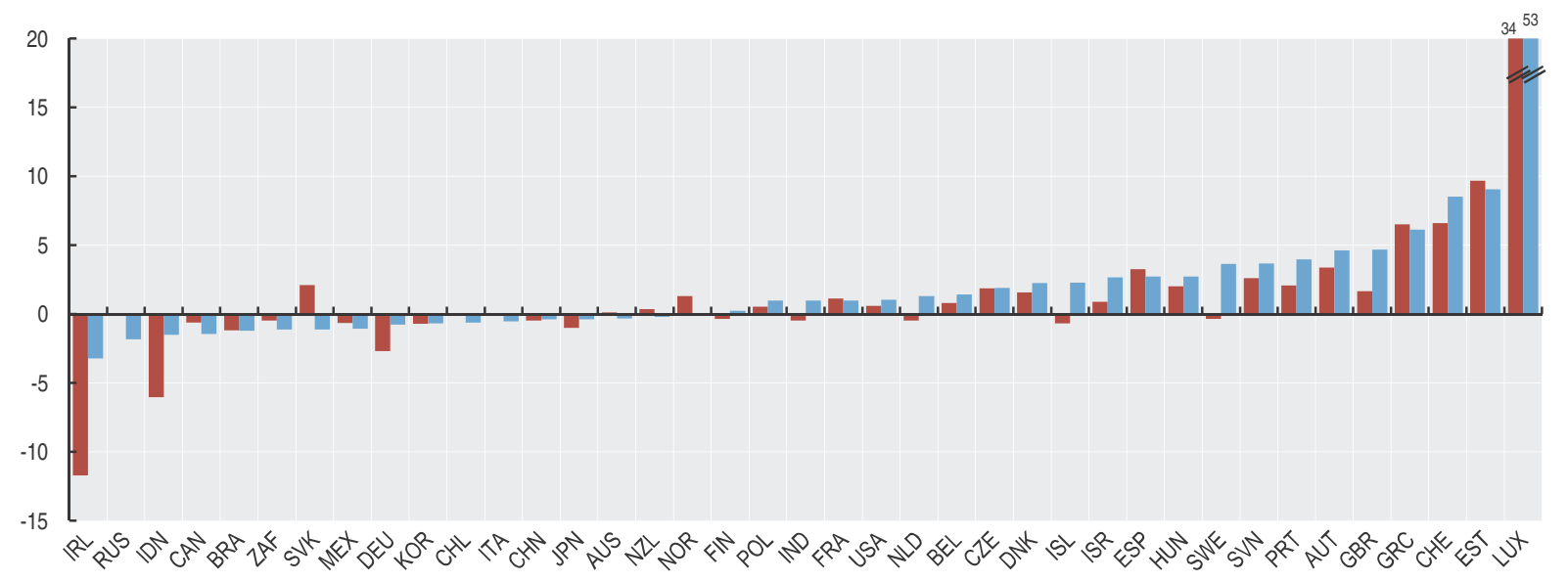




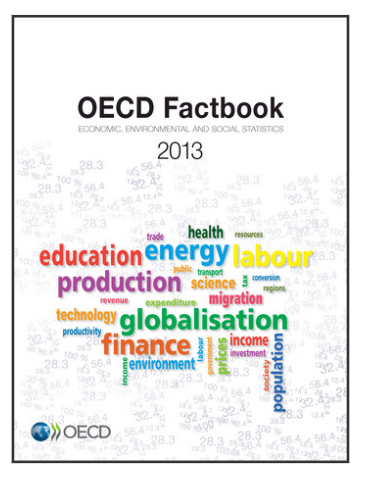

\section{From: \\ OECD Factbook 2013 \\ Economic, Environmental and Social Statistics}

Access the complete publication at:

https://doi.org/10.1787/factbook-2013-en

\section{Please cite this chapter as:}

OECD (2013), "Internationaler Handel mit Dienstleistungen", in OECD Factbook 2013: Economic, Environmental and Social Statistics, OECD Publishing, Paris.

DOI: https://doi.org/10.1787/factbook-2013-32-de

Das vorliegende Dokument wird unter der Verantwortung des Generalsekretärs der OECD veröffentlicht. Die darin zum Ausdruck gebrachten Meinungen und Argumente spiegeln nicht zwangsläufig die offizielle Einstellung der OECDMitgliedstaaten wider.

This document and any map included herein are without prejudice to the status of or sovereignty over any territory, to the delimitation of international frontiers and boundaries and to the name of any territory, city or area.

You can copy, download or print OECD content for your own use, and you can include excerpts from OECD publications, databases and multimedia products in your own documents, presentations, blogs, websites and teaching materials, provided that suitable acknowledgment of OECD as source and copyright owner is given. All requests for public or commercial use and translation rights should be submitted to rights@oecd.org. Requests for permission to photocopy portions of this material for public or commercial use shall be addressed directly to the Copyright Clearance Center (CCC) at info@copyright.com or the Centre français d'exploitation du droit de copie (CFC) at contact@cfcopies.com. 